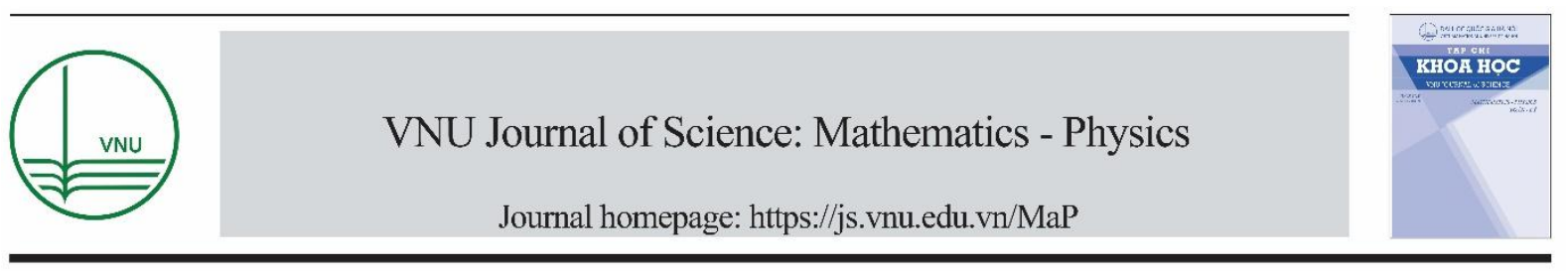

Original Article

\title{
Wave Propagation and Localization in a Complex Random Potential with Power-law Correlations: A Numerical Study
}

\author{
Ba Phi Nguyen 1,*, Huu Dinh Dang ${ }^{2}$ \\ ${ }^{I}$ Department of Basic Sciences, Mientrung University of Civil Engineering, \\ 24 Nguyen Du, Tuy Hoa, Vietnam \\ ${ }^{2}$ Department of Electrics Technology, Industrial University of Ho Chi Minh City, \\ 12 Nguyen Van Bao, Go Vap, Ho Chi Minh City, Vietnam
}

Received 19 March 2020; Accepted 25 April 2020

\begin{abstract}
In this paper, we investigate numerically wave propagation and localization in a complex random potential with power-law correlations. Using a discrete stationary Schrödinger equation with the simultaneous presence of the spatial correlation and the non-Hermiticity of the random potential in the diagonal on-site terms of the Hamiltonian, we calculate the disorderaveraged logarithmic transmittance and the localization length. From the numerical analysis, we find that the presence of power-law correlation in the imaginary part of the on-site disordered potential gives rise to the localization enhancement as compared with the case of absence of correlation. Depending on the disorder's strength, we show that there exist different behaviors of the dependence of the localization on the correlation strength.

Keywords: Non-Hermitian Hamiltonian, complex disordered potential, spatial correlated disorder, Anderson localization.
\end{abstract}

\section{Introduction}

Although Anderson localization of quantum particles and classical waves has been extensively studied over the last 60 years, it is still attracting the interest of many researchers in various areas of physics [1-6]. New aspects of localization phenomena have been discovered continually in diverse systems characterized by different kinds of wave equations or random potentials. Among them, the combined influence of the spatial correlation and the non-Hermiticity of the random potential is the main focus of this study.

\footnotetext{
${ }^{*}$ Corresponding author.

Email address: nguyenbaphi@muce.edu.vn
}

https//doi.org/ 10.25073/2588-1124/vnumap.4498 
During the recent few decades, the influence of spatially correlated disorder on the wave propagation and localization properties has been attracted much attention from theoretical as well as experimental researchers [7-23]. It has been demonstrated that some special kinds of short-range correlated disorder can produce delocalized eigenstates even in one dimension [7, 8]. More interestingly, it was predicted that the introduction of long-range correlations into a disordered system would suppress localization and give rise to a continuum of delocalized states [9, 10]. At first, this prediction caused some controversy $[9,11]$. However, it was experimentally verified later by a measurement of microwave transmission spectra through a single-mode waveguide with artificially introduced correlated disorder [12]. There is a common point of view that the strongest localization is obtained for strongly disordered uncorrelated potentials, which is based on the prediction that correlations suppress, rather than enhance, localization. In some specific situations [24-27], however, a strong decrease of the localization length and therefore a strong enhancement of localization has been observed, when the long-range correlated disorder is introduced into the Hermitian Hamiltonian system. In spite of a large number of publications devoted to this topic, the problem of understanding the effects of spatial correlations on Anderson localization has not been completely resolved yet.

In recent years, there has been considerable attention devoted to studying transport and localization properties in physical systems which are described by non-Hermitian Hamiltonians [2839]. These kinds of Hamiltonians are usually regarded as effective Hamiltonians for which the nonHermitian part can serve for different purposes. The possibility for the potential to possess an imaginary part makes these non-Hermitian Hamiltonians having many unique features such as nonHermitian delocalization transition [28, 29], a transition from ballistic to diffusive transport [30], oneway scattering and transport $[33,34]$ and topological phase transitions $[35,36]$. Basically, there are two classes of problems associated with non-Hermitian Hamiltonian: one in which the non-Hermitcity arises from the off-diagonal elements such as the Hatano-Nelson model, where an imaginary vector potential is applied to the Hamiltonian [28] and the other in which the non-Hermitcity originates from the complexity of on-site potentials [31, 37-39]. For the latter, a one-dimensional (1D) non-Hermitian lattice model with randomness only in the imaginary part of the on-site potential has been studied, with the result that the eigenstates of such a model are localized, but the properties of localization are attributed to be qualitatively different from those of the usual Anderson model [31]. The 2D nonHermitian square lattice model was proposed in a recent work by Tzortzakakis and co-workers [38]. They demonstrated that the complex disordered potential acts in a dual way: its time-reversal symmetry breaking favors more delocalized states, while its disorder nature favors more localized ones. Very shortly after, Huang and Shklovskii extended the 2D square lattice model to 3D cubic one [39]. The authors showed that there exists a transition from delocalized to localized states as in the usual 3D Anderson model. However, this transition happens at a substantially lower critical value of the disorder strength than that of the Hermitian case $\left(W_{c}=6.1\right.$ vs $\left.W_{c}=16.5\right)$.

One important application of Anderson localization in optical systems is to develop random lasers [40, 41], where an optical cavity is formed not by mirrors but by multiple scattering in a random gain medium. To achieve high-efficiency output from a random laser, it is crucial to produce strongly localized states. To do this, in our very recent work [42], we studied the effects of spatial correlations on the transverse localization of waves in 1D lattices with randomly distributed gain and loss. In the short-ranged case and when the correlation length is sufficiently small, we find that there exists a critical value of the disorder strength, below which enhancement and above which suppression of localization occurs as the correlation length increases. In the region where the correlation length is larger, localization is suppressed in all cases. Similar behavior is obtained for long-range correlations as the disorder strength and the correlation exponent are varied. In the region where localization is 
enhanced in the presence of long-range correlations, we find that the enhancement occurs in the whole energy band, but is strongest near the band center.

In this paper, we study the effects of power-law correlations on the transmission and the longitudinal localization of plane waves in a $1 \mathrm{D}$ disordered lattice chain characterized by a random non-Hermitian Hamiltonian, where the imaginary part of the on-site potential is a correlated random function of the position. Using a calculation scheme for solving a disordered version of the stationary discrete Schrödinger equation, we calculate the disorder-averaged logarithmic transmittance and the localization length, when the intensity of the incident wave is held fixed. From the numerical results, we find that the presence of power-law correlation in the disorder distribution gives rise to the localization enhancement as compared with the case of absence of correlation. Specifically, in the region where the correlation is relatively weak, it is shown that the localization is enhanced rapidly with increasing the correlation strength. In the region where the disorder strength is weak, a nonmonotonic dependence of the localization length on the correlation strength is found. This behavior is held in the entire energy band but is more pronounced at the band center than near the band edges. In the region where the disorder strength is large enough, the localization length is a monotonic decreasing function of the correlation strength.

The rest of this paper is organized as follows. In section 2, we describe the theoretical model and the method within the nearest-neighbor tight-binding approximation. The physical quantities of interest are also described briefly in this section. In section 3, we present our numerical results. Finally, in section 4, we conclude the paper.

\section{Theoretical Model and Method}

\subsection{Model}

We are interested in the stationary propagation of extended excitations through a 1D lattice chain of length $L$ described by a discrete stationary Schrödinger equation

$$
\varepsilon_{n} \psi_{n}+J\left(\psi_{n-1}+\psi_{n+1}\right)=E \psi_{n}
$$

This is an open quantum system which is coupled to the external world through two identical semi-infinite perfect leads on either side. Here $\varepsilon_{n}$ is the wave function amplitude at the $n$th lattice site, $E$ is the energy of the incident wave, and $J$ is the constant nearest-neighbor hopping term. It is wellknown that when the on-site potential $\varepsilon_{n}$ are assumed to be real, random, uncorrelated, and uniformly distributed in the interval $[-W / 2, W / 2]$ with $W$ being the disorder strength, we have the usual Anderson model [1].

In the present work, the on-site potential $\varepsilon_{n}$ is considered to be complex. Specifically, it takes the form,

$$
\varepsilon_{n}=\varepsilon_{n}^{R}+i \varepsilon_{n}^{I}
$$

where the real part $\varepsilon_{n}^{R}$ is assumed to be the same for all lattice sites (below we put $\varepsilon_{n}^{R}=0$ for simplicity), whereas the imaginary part $\varepsilon_{n}^{I}$ is considered to be a sequence of random variables with power-law correlations. One of the most popular ways to numerically generate such a sequence is to consider the trace of the fractional Brownian motion defined by $[9,19]$ 


$$
\gamma_{n}=\sum_{k=1}^{L / 2}\left[k^{-\alpha}\left(\frac{2 \pi}{L}\right)^{(1-\alpha)}\right]^{1 / 2} \cos \left(\frac{2 \pi n k}{L}+\phi_{k}\right)
$$

where where the number of sites $L$ is an even number and $\phi_{k}$ 's are $L / 2$ random phases uniformly distributed in the interval $[0,2 \pi]$. The Fourier transform of the two-point correlation function $\left\langle\gamma_{i} \gamma_{j}\right\rangle$, $S(k)$, is proportional to a power-law spectrum $k^{-\alpha}$. The exponent $\alpha$ determines the roughness of potential landscapes and characterizes the power-law correlation strength. When $\alpha$ is zero, we recover an uncorrelated random sequence. In order to study the dependence on the strength of disorder properly, we need to normalize the generated sequence $\gamma_{n}$ by multiplying a suitable normalization constant [14]

$$
\varepsilon_{n}^{I}=\frac{W}{\sqrt{12}} \frac{\gamma_{n}}{C}
$$

where $W$ measures the disorder strength and $C$ is the normalization constant which is obtained by the condition $\sqrt{\left\langle\gamma_{n}^{2}\right\rangle-\left\langle\gamma_{n}\right\rangle^{2}}=1$. In Figure 1, we show an example of typical random configurations of $\varepsilon_{n}^{I}$ corresponding to $W=2.0$ and $\alpha=0,1.5,2.5$. It is clearly that the normalization effectively modifies the local disorder strength while keeping the global disorder strength the same for any $\alpha$.

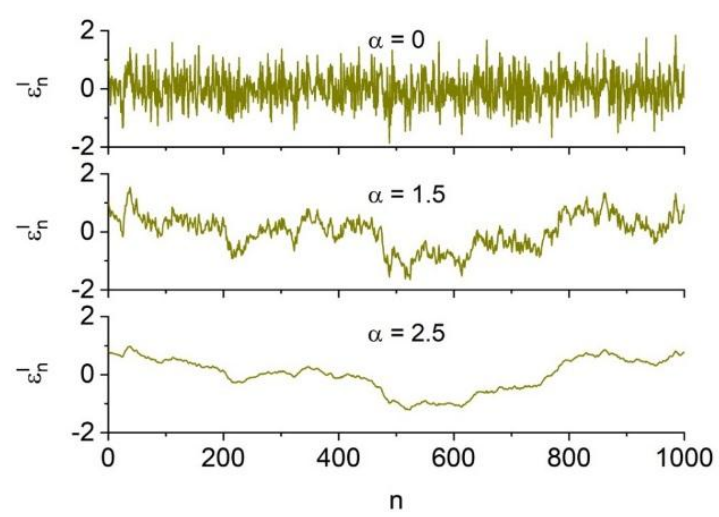

Figure 1. Typical random configurations of $\varepsilon_{n}^{I}$, when $W=2.0$ and the power-law correlation exponent $\alpha=0,1.5,2.5$. It is clearly that the normalization effectively modifies the local disorder strength while keeping the global disorder strength the same for different values of $\alpha$.

\subsection{Method}

We assume that a plan wave is incident from the right onto the disordered sample of L lattice sites and define the transmission problem by using

$$
\begin{cases}\psi_{n+1}=\left(E-\varepsilon_{n}\right) \psi_{n}-\psi_{n-1}, & 0<n<L \\ \psi_{n}=r_{0} e^{i q(L-n)}+r_{1} e^{i q(n-L)}, & n \geq L \\ \psi_{n}=t e^{-i q n}, \quad n \leq 0 & \end{cases}
$$


where the wave number $q$ is related to the energy of the incident wave $E$ by $E=2 \cos q ; r_{0}, r_{1}$ and $t$ are the complex amplitudes of the incident, reflected and transmitted waves, respectively. Since our model is non-Hermitian, the conservation of probability current is not satisfied, therefore $\left|r_{1}\right|^{2}+|t|^{2} \neq\left|r_{0}\right|^{2}$. The wave number $q$ is assumed to be the same inside and outside the medium. This restriction corresponds to the case where the creation of other harmonics is neglected.

For the transmission problem, in principle, there are two cases for which we need to be carefully distinguished. The case where the value of $|t|^{2}$ is fixed is called the fixed output case, while that with a fixed value of $\left|r_{0}\right|^{2}$ is called the fixed input case. In actual experiments, however, one usually fixes the incident wave intensity while varying other parameters. Therefore, we developed a reliable theoretical method for solving Eqs. (4) and (5) numerically in the situation where the incident wave intensity, $\left|r_{0}\right|^{2}$, is fixed [43-45]. In particular, we first assume that $t$ is a certain positive real number. Using the relationships $\psi_{-1}=t e^{i q}, \psi_{0}=t$ and $\psi_{1}=E \psi_{0}-\psi_{-1}$ and solving the first of Eq. (5) iteratively, we obtain $\psi_{L}$ and $\psi_{L+1}$ for a given $L$. Next, using the relationships $\psi_{L}=r_{0}+r_{1}$ and $\psi_{L+1}=r_{0} e^{-i q}+r_{1} e^{i q}$, we express $r_{0}$ and $r_{1}$ as

$$
r_{0}=\frac{\psi_{L} e^{i q}-\psi_{L+1}}{e^{i q}-e^{-i q}} \text { and } r_{1}=\frac{\psi_{L+1}-\psi_{L} e^{-i q}}{e^{i q}-e^{-i q}}
$$

And then the expressions for the transmittance $T$ and the reflectance $R$ are given by

$$
T=\left|\frac{t}{r_{0}}\right|^{2}=|t|^{2} \frac{4 \sin ^{2} q}{\left|\psi_{L} e^{i q}-\psi_{L+1}\right|^{2}} \text { and } R=\left|\frac{r_{1}}{r_{0}}\right|^{2}=\frac{\left|\psi_{L} e^{-i q}-\psi_{L+1}\right|^{2}}{\left|\psi_{L} e^{i q}-\psi_{L+1}\right|^{2}}
$$

In order to calculate $T$ and $R$ in the fixed input problem, we first choose the values of $E$ (or $q$ ), $\left|r_{0}\right|^{2}$ and a random configuration of $\varepsilon_{n}$. Then we solve Eq. (1) repeatedly for many different initial values of $t$ $(t=0, \delta, 2 \delta, 3 \delta, \ldots)$ until we obtain the final value of $\left|r_{0}\right|^{2}$ sufficiently close to the chosen value. The step size $\delta$ needs to be chosen appropriately to achieve desired accuracy.

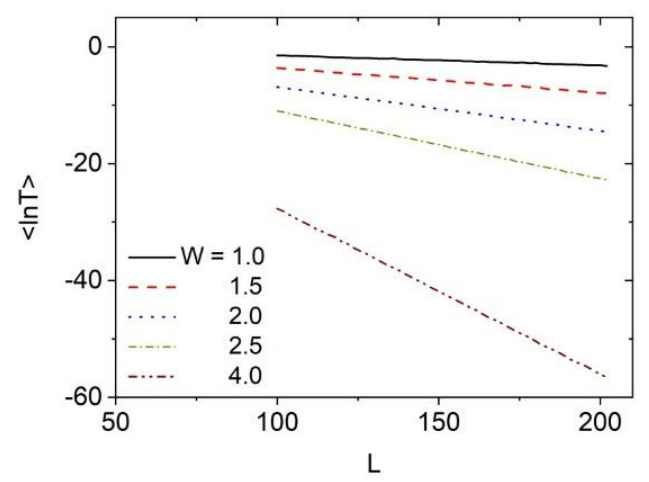

Figure 2. In the absence of power-law correlation $(\alpha=0)$ : Disorder-averaged logarithmic transmittance $\langle\ln T\rangle$ versus $L$ at $E=0$ for various values of the disorder strength $W$. 
In the study of the Anderson localization phenomenon of waves in disordered media, the localization length $\xi$ is the central quantity which is defined by [2]

$$
\lim _{L \rightarrow \infty}\langle\ln T\rangle=-\frac{L}{\xi}
$$

where $\langle\ldots\rangle$ denotes averaging over a large number of random configurations of $\varepsilon_{n}$. The localization length measures the exponential decay rate of a localized state in the thermodynamic limit ( $L \rightarrow \infty$ or $T \rightarrow 0$ ).

\section{Numerical Results}

In our calculations, the disorder-averaged quantities are obtained by averaging over $10^{4}$ distinct disorder configurations. Although the chosen system sizes are finite, they are large enough so that the asymptotic behaviors in the thermodynamic limit should be achieved. Indeed, as seen below that the exponential decay of $\langle T\rangle$ (or the linear decay of $\langle\ln T\rangle$ ) with $L$ approaches to some very small values $(\langle T\rangle \rightarrow 0$ ) when the calculations were performed for the system size $L=100$ up to $L=200$. All of our results were obtained for the fixed input intensity of $\left|r_{0}\right|^{2}=1$. The step size for $t$ was $\delta=10^{-6}$. The error in the calculated value of $\left|r_{0}\right|^{2}=1$ was smaller than $10^{-5}$.

First, we consider the problem in the case of absence of power-law correlation. In Figure 2, we plot $\langle\ln T\rangle$ versus $L$ at the band center $(E=0)$ for various values of the disorder strength $W$ when $\alpha=0$. From the numerical results we find that for a given $W,\langle\ln T\rangle$ decays linearly as $L$ increases to infinity. This is a signature of Anderson localization. We have also verified and found that this behavior is held for all values of $E$ in the entire energy band. Similarly to the usual Anderson model where the real part of the on-site potential is random, in the model under consideration, the decay rate of $\langle\ln T\rangle$ increases, hence the localization is enhanced as the disorder strength $W$ increases. These are in good agreement with the results of previous studies [31, 37-39], in which the transverse localization of waves in 1D, 2D and 3D optical waveguide arrays with non-Hermitian disorder has been investigated in details.

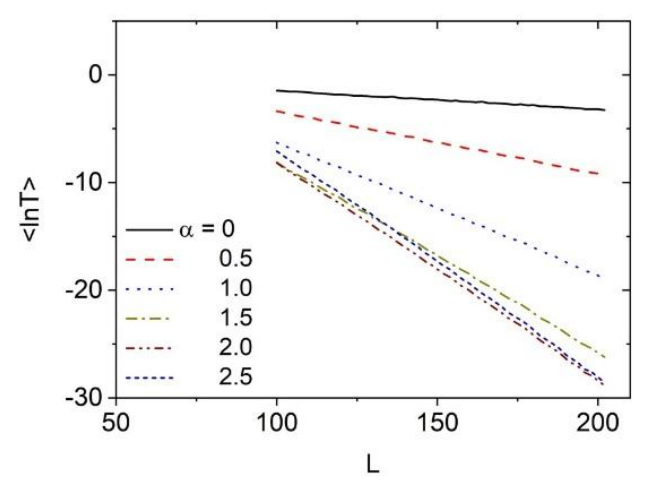


Figure 3. In the presence of power-law correlation $(\alpha \neq 0)$ : Disorder-averaged logarithmic transmittance $\langle\ln T\rangle$ versus $L$ at $E=0$ for various different values of $\alpha$ when $W=1.0$.

Next, we study the effects of spatially correlated disorder on the longitudinal localization of waves for which the amplitude of the wave decays exponentially along the propagation direction. This is the main goal of the present work. In Figure 3, we plot $\langle\ln T\rangle$ versus $L$ at $E=0$ when $W=1.0$ for various values of the power-law correlation exponent $\alpha$. We find that in the presence of power-law correlation, the linear decay of $\langle\ln T\rangle$ with $L$ is maintained. The decay rate of $\langle\ln T\rangle$ is increased, hence the behavior of localization is enhanced as $\alpha$ increases. Remarkably, in the correlation region with $\alpha<2,\langle\ln T\rangle$ decays more and more rapidly with $L$ as $\alpha$ increases. However, if we keep increasing $\alpha$ further, the decay rate of $\langle\ln T\rangle$ slows down and stops at a certain critical value $\alpha_{c}$.

The next question we ask is how this behavior changes as $\alpha$ is increased beyond the critical value of $\alpha_{c}$. The answer is shown clearly in Figure 4, which plots the inverse localization length $1 / \xi$ as a function of the correlation parameter $\alpha$ at $E=0$ for several different values of the disorder strength $W$. In the parameter region under consideration, we find that the behavior of localization is always enhanced in the case of $\alpha \neq 0$ as compared with the case of $\alpha=0$. When $W$ is small, we find that the inverse localization length $1 / \xi$ shows a non-monotonic dependence on $\alpha$. Particularly, in the region where $\alpha$ is relatively small, $1 / \xi$ increases rapidly with increasing $\alpha$. However, increasing $1 / \xi$ with $\alpha$ will slow down and $1 / \xi$ attains a maximum value at a critical value $\alpha_{c}$. If we continue increasing $\alpha$ further, $1 / \xi$ returns to decrease slowly with $\alpha$. This implies that the correlation-induced localization enhancement is the strongest at $\alpha_{c}$. It is clearly seen that the value of $\alpha_{c}$ strongly depends on the disorder strength $W$, specifically, $\alpha_{c}$ increases with increasing $W$. As a result, when $W$ is sufficiently large ( $W>4$ : bandwidth), the localization length $\xi$ would be a monotonic decreasing function of $\alpha$. These results are completely consistent with those presented in our recent work [42], in which the effects of spatially correlated disorder on the transverse localization of waves in a 1D array of optical waveguides have been studied.

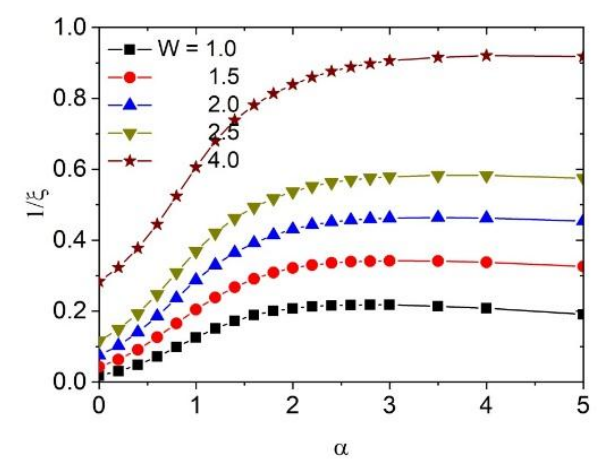

Figure 4. Inverse localization length $1 / \xi$ versus power-law correlation strength $\alpha$, when $E=0$ for various values of the disorder strength $W$. 
Finally, in Figure 5, we depict the inverse localization length $1 / \xi$ as a function of the correlation parameter $\alpha$ at $W=1.0$ for three typical values of the energy of the incident wave, $E=0,1$ and $\sqrt{3}$. We find that the behavior of non-monotonic dependence of the inverse localization length on the correlation strength is held in the whole energy band. It appears to be more pronounced at the band center than near the band edges.

It is important to mention that although it is not presented in this paper, we have checked numerically that all the results reported above are also valid in the situations where the average value of the imaginary part of the on-site potential $\varepsilon_{n}^{I}$ takes any nonzero value. These include the cases where $\varepsilon_{n}^{I}$ takes only positive or negative random values.

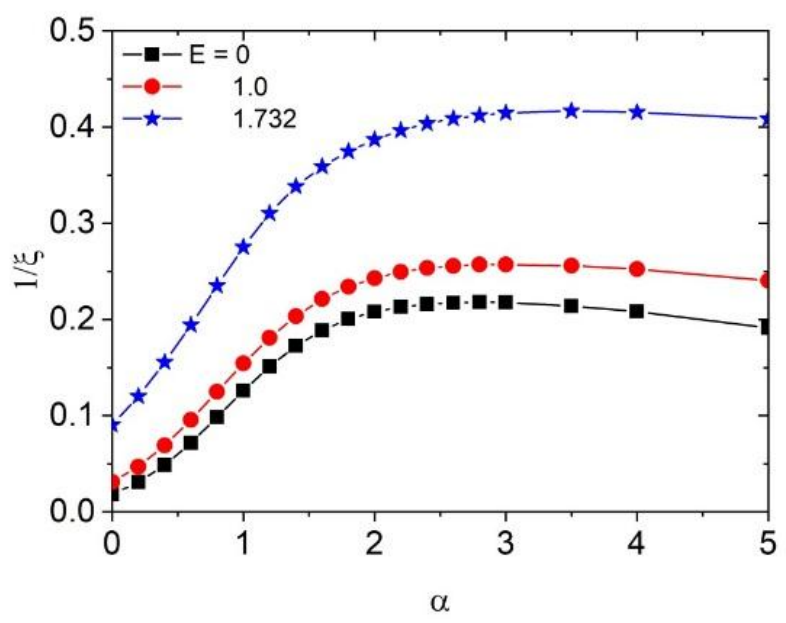

Figure 5. Inverse localization length $1 / \xi$ versus power-law correlation strength $\alpha$, when $W=1.0$ for several typical values of the incident wave energy $E$.

\section{Conclusion}

In summary, we have studied the effects of power-law correlations on the transmission and the Anderson localization of plane waves in a 1D disordered lattice chain characterized by a random nonHermitian Hamiltonian, where the imaginary part of the on-site potential is a correlated random function of the position. Using a calculation scheme for solving a disordered version of the stationary discrete Schrödinger equation, we have calculated the disorder-averaged logarithmic transmittance and the localization length, when the intensity of the incident wave is held fixed. From the numerical results, we have found that the presence of power-law correlation in the disorder distribution gives rise to the localization enhancement as compared with the case of absence of correlation. Specifically, in the region where the correlation is relatively weak, we have found that the localization is enhanced rapidly with increasing the correlation strength. In the region where the disorder strength is weak $(W<4)$, a non-monotonic dependence of the localization length on the correlation strength has been found. This behavior is held in the entire energy band but is more pronounced at the band center than near the band edges. In the region where the disorder strength is large enough $(W>4)$, the localization length is a monotonic decreasing function of the correlation strength. Devices such as random lasers utilize 
highly disordered gain materials. Our results can provide a useful guideline for achieving correlationenhanced localized states in such structures, thereby enhancing the device efficiency.

\section{Acknowledgments}

This research is funded by Vietnam National Foundation for Science and Technology Development (NAFOSTED) under Grant No. 103.01-2018.05.

\section{References}

[1] P.W. Anderson, Absence of diffusion in certain random lattices, Phys. Rev. 109 (1958) 1492-1505. https://doi.org/10.1103/PhysRev.109.1492.

[2] E. Abrahams, 50 years of Anderson localization, World Scientific, Singapore, 2010.

[3] S.A. Gredeskul, Y.S. Kivshar, A.A. Asatryan, K.Y. Bliokh, Y.P. Bliokh, V.D. Freilikher, I.V. Shadrivov, Anderson localization in metamaterials and other complex media, Low. Temp. Phys. 38 (2012) 570-602. https://doi.org/10.1063/1.4736617.

[4] M. Segev, Y. Silberberg, D.N. Christodoulides, Anderson localization of light, Nat. Photon. 197 (2013) 197-204. https://doi.org/10.1038/nphoton.2013.30.

[5] H.H. Sheinfux, Y. Lumer, G. Ankonina, A.Z. Genack, G. Bartal, M. Segev, Observation of Anderson localization in disordered nanophotonic structures, Science 356 (2017) 953-956. https://science.sciencemag.org/content/356/6341/953

[6] S. Kim, K. Kim, Anderson localization and delocalization of massless two-dimensional Dirac electrons in random one-dimensional scalar and vector potentials, Phys. Rev B. 99 (2019) 014205. https://doi.org/10.1103/PhysRevB.99.014205.

[7] D.H. Dunlap, H.L. Wu, P.W. Phillips, Absence of localization in a random-dimer model, Phys. Rev. Lett. 88 (1990) 88-91. https://doi.org/10.1103/PhysRevLett.65.88.

[8] V. Bellani, E. Diez, R. Hey, L. Toni, L. Tarricone, G.B. Parravicini, F. Domínguez-Adame, R. Gómez-Alcalá, Experimental evidence of delocalized states in random dimer superlattices, Phys. Rev. Lett. 82 (1999) 2159-2162. https://doi.org/10.1103/PhysRevLett.82.2159.

[9] F.A.B. F de Moura, M.L. Lyra, Delocalization in the 1D Anderson model with long-range correlated disorder, Phys. Rev. Lett. 81 (1998) 3735-3738. https://doi.org/10.1103/PhysRevLett.81.3735.

[10] F.M. Izrailev, A.A. Krokhin, Localization and the mobility edge in one-dimensional potentials with correlated disorder, Phys. Rev. Lett. 82 (1999) 4062-4065. https://doi.org/10.1103/PhysRevLett.82.4062.

[11] J.M. Kantelhardt, S. Russ, A. Bunde, S. Havlin, I. Webman, Comment on "Delocalization in the 1D Anderson model with long-range correlated disorder", Phys. Rev. Lett. 84 (2000) 198. https://doi.org/10.1103/PhysRevLett.84.198.

[12] U. Kuhl, F.M. Izrailev, A.A. Krokhin, and H.J. Stöckmann, Experimental observation of the mobility edge in a waveguide with correlated disorder, Appl. Phys. Lett. 77 (2000) 633-635. https://doi.org/10.1063/1.127068.

[13] H. Shima, T. Nomura, T. Nakayama, Localization-delocalization transition in one-dimensional electron systems with long-range correlated disorder, Phys. Rev. B 70 (2004) 075116. https://doi.org/10.1103/PhysRevB.70.075116.

[14] T. Kaya, Localization-delocalization transition in chains with long-range correlated disorder, Eur. Phys. J. B 55 (2007) 49-56. https://doi.org/10.1140/epjb/e2007-00036-4.

[15] S. Nishino, K. Yakubo, H. Shima, Finite size effects in infinitely large electronic systems with correlated disorders, Phys. Rev. B 79 (2009) 033105. https://doi.org/10.1103/PhysRevB.79.033105.

[16] A.M. García-García, E. Cuevas, Differentiable potentials and metallic states in disordered one-dimensional systems, Phys. Rev. B 79 (2009) 073104. https://doi.org/10.1103/PhysRevB.79.073104.

[17] L.Y. Gong, P.Q. Tong, Z.C. Zhou, von Neumann entropy signatures of a transition in one-dimensional electron systems with long-range correlated disorder, Eur. Phys. J. B 77 (2010) 413-417. https://doi.org/10.1140/epjb/e2010-00283-2. 
[18] A. Croy, P. Cain, M. Schreiber, Anderson localization in 1D systems with correlated disorder, Eur. Phys. J. B 82 (2011) 107-112. https://doi.org/10.1140/epjb/e2011-20212-1.

[19] B.P. Nguyen, K. Kim, Influence of weak nonlinearity on the 1D Anderson model with long-range correlated disorder, Eur. Phys. J. B 84 (2011) 79-82. https://doi.org/10.1140/epjb/e2011-20608-9.

[20] F.M. Izrailev, A.A. Krokhin, N.M. Makarov, Anomalous localization in low-dimensional systems with correlated disorder, Phys. Rep. 512 (2012) 125-254. https://doi.org/10.1016/j.physrep.2011.11.002.

[21] G.M. Petersen, N. Sandler, Anticorrelations from power-law spectral disorder and conditions for an Anderson transition, Phys. Rev. B 87 (2013) 195443. https://doi.org/10.1103/PhysRevB.87.195443.

[22] W. Choi, C. Yin, I.R. Hooper, W.L. Barnes, J. Bertolotti, Absence of Anderson localization in certain random lattices, Phys. Rev. E 96 (2017) 022122. https://doi.org/10.1103/PhysRevE.96.022122.

[23] J.R.F. Lima, L.F.C. Pereira, A.L.R. Barbosa, Dirac wave transmission in Levy disordered systems, Phys. Rev. E 99 (2019) 032118. https://doi.org/10.1103/PhysRevE.99.032118.

[24] U. Kuhl, F.M. Izrailev, A.A. Krokhin, Enhancement of localization in one-dimensional random potentials with long-range correlations, Phys. Rev. Lett. 100 (2008) 126402. https://doi.org/10.1103/PhysRevLett.100.126402.

[25] W. Zhao, J.W. Ding, Enhanced localization and delocalization in surface disordered quantum waveguides with long-range correlation, EPL. 89 (2010) 57005. https://doi.org/10.1209/0295-5075/89/57005.

[26] C.S. Deng, H. Xu, Anomalous localization and dual role of correlation in one-dimensional electronic systems with long-range correlated disorder, Physica E 44 (2012) 1747-1751. https://doi.org/10.1016/j.physe.2011.12.002.

[27] M.K. Nezhad, S.M. Mahdavi, A.R. Bahrampour, M. Golshani, Effect of long-range correlated disorder on the transverse localization of light in 1D array of optical waveguides, Opt. Commun. 307 (2013) 39-45. https://doi.org/10.1016/j.optcom.2013.06.004.

[28] N. Hatano, D.R. Nelson, Localization transitions in non-Hermitian quantum mechanics, Phys. Rev. Lett. 77 (1996) 570-573. https://doi.org/10.1103/PhysRevLett.77.570.

[29] A. V. Kolesnikov, K.B. Efetov, Localization-delocalization transition in non-Hermitian disordered systems, Phys. Rev. Lett. 84 (2000) 5600. https://doi.org/10.1103/PhysRevLett.84.5600.

[30] T. Eichelkraut, R. Heilmann, S. Weimann, S. Stützer, F. Dreisow, D.N. Christodoulides, S. Nolte, A. Szameit, Mobility transition from ballistic to diffusive transport in non-Hermitian lattices, Nat. Commun. 4 (2013) 3533. https://doi.org/10.1038/ncomms3533.

[31] A. Basiri, Y. Bromberg, A. Yamilov, H. Cao, T. Kottos, Light localization induced by a random imaginary refractive index, Phys. Rev. A 90 (2014) 043815. https://doi.org/10.1103/PhysRevA.90.043815.

[32] B.P. Nguyen, K. Kim, Transport and localization of waves in ladder-shaped lattices with locally PT-symmetric potentials, Phys. Rev. A 94 (2016) 062122. https://doi.org/10.1103/PhysRevA.94.062122.

[33] S. Longhi, Bloch oscillations in complex crystals with PT symmetry, Phys. Rev. Lett. 103 (2009) 123601. https://doi.org/10.1103/PhysRevLett.103.123601.

[34] S. Longhi, D. Gatti, G. Della Valle, Non-Hermitian transparency and on-way transport in low-dimensional lattices by an imaginary gauge field, Phys. Rev. B 92 (2015) 094204. https://doi.org/10.1103/PhysRevB.92.094204.

[35] M.S. Rudner, L.S. Levitov, Topological transition in a non-Hermitian quantum walk, Phys. Rev. Lett. 102 (2009) 065703. https://doi.org/10.1103/PhysRevLett.102.065703.

[36] J.M. Zuener, M.C. Rechtsman, Y. Plotnik, Y. Lumer, S. Nolte, M.S. Rudner, M. Segev, A. Szameit, Observation of a topological transition in the bulk of a non-Hermitian system, Phys. Rev. Lett. 115 (2015) 040402. https://doi.org/10.1103/PhysRevLett.115.040402.

[37] B.P. Nguyen, D.K. Phung, K. Kim, Anomalous localization enhancement in one-dimensional non-Hermitian disordered lattices, J. Phys. A: Math. Theor. 53 (2020) 045003. https://doi.org/10.1088/1751-8121/ab5eb8.

[38] A.F. Tzortzakakis, K.G. Makris, E.N. Economou, Non-Hermitian disorder in two-dimensional optical lattices, Phys. Rev. B 101 (2020) 014202. https://doi.org/10.1103/PhysRevB.101.014202.

[39] Y. Huang, B.I. Shklovskii, Anderson transition in three-dimensional systems with non-Hermitian disorder, Phys. Rev. B 101 (2020) 014204. https://doi.org/10.1103/PhysRevB.101.014204.

[40] D.S. Wiersma, The physics and applications of random lasers, Nat. Phys. 4 (2008) 359-367. https://doi.org/10.1038/nphys971. 
[41] B. Abaie, E. Mobini, S. Karbasi, T. Hawkins, J. Ballato, A. Mafi, Random lasing in an Anderson localizing optical fiber, Light Sci. Appl. 6 (2016) e17041. https://doi.org/10.1038/lsa.2017.41.

[42] B.P. Nguyen, T.K.T. Lieu, K. Kim, Numerical study of the transverse localization of waves in one-dimensional lattices with randomly distributed gain and loss: Effect of disorder correlations, Wave Random Complex Media, https://doi.org/10.1080/17455030.2020.1774680.

[43] B.P. Nguyen, K. Kim, F. Rotermund, H. Lim, Enhanced localization of waves in one-dimensional random media due to nonlinearity: Fixed input case, Physica B 406 (2011) 4535. https://doi.org/10.1016/j.physb.2011.09.034.

[44] B.P. Nguyen, K. Kim, Transmission, reflection and localization of waves in one-dimensional amplifying media with nonlinear gain, J. Korean Phys. Soc. 64 (2014) 1665. https://doi.org/10.3938/jkps.64.1665.

[45] B.P. Nguyen, K. Kim, Anderson localization and saturable nonlinearity in one-dimensional disordered lattices, J. Mod. Opt. 64 (2017) 1923-1927. https://doi.org/10.1080/09500340.2017.1326639. 